02,13

\title{
Метод расчета критического тока неоднородных сверхпроводящих пленок
}

\author{
(С) П.И. Безотосный, С.Ю. Гаврилкин, К.А. Дмитриева, А.Н. Лыков, А.Ю. Цветков \\ Физический институт им. П.Н. Лебедева РАН, \\ Москва, Россия \\ E-mail: tsvetkov@sci.lebedev.ru
}

(Поступила в Редакцию 20 августа 2018 г.)

\begin{abstract}
Предложен метод расчета критического тока неоднородной сверхпроводящей пленки (пластины) при различных значениях внешнего магнитного поля. Изменение сверхпроводящих свойств задается путем варьирования значений длины когерентности и лондоновской глубины проникновения по толщине. При этом длина когерентности максимальна в центре пластины и убывает при приближении к ее границам, а лондоновская глубина, наоборот, достигает минимума в центре пластины и возрастает при приближении к ее границам. В рамках предложенного подхода проведены расчеты и сравнение зависимостей критического тока от внешнего магнитного поля для двух случаев: неоднородного и однородного распределений сверхпроводящих свойств по толщине пленки. Установлено, что при неоднородном распределении сверхпроводящих свойств значения критического тока пластины больше, чем при однородном случае при таких же значениях внешнего магнитного поля. Это связано с тем, что параметр порядка перераспределяется в неоднородной пластине по толщине таким образом, что сверхпроводящее состояние становится более устойчивым к влиянию тока и магнитного поля. Показано, что безвихревое мейснеровское состояние в неоднородных пленках сохраняется при более высоких полях по сравнению с однородными пленками. При этом, чем больше неоднородность пленки, тем мейснеровское состояние устойчивее к влиянию внешнего магнитного поля.
\end{abstract}

DOI: 10.21883/FTT.2019.02.47119.239

\section{1. Введение}

Тонкие сверхпроводящие пленки (пластины) нередко являются объектами исследования фундаментальных свойств сверхпроводящих материалов [1]. Информацию, касающуюся микроскопической структуры сверхпроводника, можно получить с помощью измерений его макроскопических параметров (таких как проводимость, теплоемкость, намагниченность). При этом величины параметров, в частности критических, сверхпроводящих пленок отличаются от значений для объемного материала. Свойства пленок во многом определяются границами раздела между материалом подложки и сверхпроводником, а также между сверхпроводником и внешней средой. Для описания фундаментальных свойств материала, не зависящих от геометрической формы образца, необходимы модели, описывающие объект как совокупность его объемных и геометрических свойств. Особенностью тонких пленок является неоднородность их свойств (если не принять специальных мер), в том числе сверхпроводящих, по толщине. Например, подложка и сама пленка обладают различными периодами решетки, что приводит к наличию переходного слоя между ними. Длина свободного пробега электронов в этом слое меньше, чем в объеме сверхпроводника. Если пленка достаточно тонкая, параметры ее решетки, а также другие свойства могут отличаться от свойств материала, из которого сделана пленка. Поэтому модели для описания реальных сверхпроводящих пленок должны учитывать неоднородность свойств по их толщине.
Ранее в рамках теории Гинзбурга-Ландау (ГЛ) было проведено теоретическое исследование свойств сверхпроводящих пленок [2-4]. Согласно полученным результатам, для пластин толщиной меньше или порядка длины когерентности $\xi$ влияние границы на их сверхпроводящие свойства существенно. Аналитические формулы, описывающие сверхпроводящее состояние тонких пленок, как правило, выведены для случая пленки толщиной много меньше как $\xi$, так и лондоновской глубины проникновения магнитного поля $\lambda$. Для изучения свойств пленок толщиной порядка $\xi$ и $\lambda$ были использованы численные методы.

В рамках настоящей работы предложен алгоритм описания свойств сверхпроводящих пластин, который принимает в расчет неоднородность сверхпроводящих свойств по их толщине. Используя данный метод, были рассчитаны и проанализированы зависимости критического тока от магнитного поля, параллельного поверхности.

\section{2. Описание модели}

Расчет критического тока сверхпроводящих пленок осуществлялся численными методами в рамках теории ГЛ. В данном случае рассматривалась длинная и широкая сверхпроводящая пластина толщиной $D$ (длина и ширина много больше $D$ ) в магнитном поле $\mathbf{H}$, параллельном ее поверхности. Используется декартова система координат $(x, y, z)$ с осями $y$ и $z$, направленными параллельно плоскости поверхности пленки, 
причем ось $z$ направлена параллельно внешнему магнитному полю, а транспортный ток течет вдоль оси $y$. Используя обычный метод выбора калибровки векторпотенциала А, можно записать уравнения ГЛ в следующем виде:

$$
\begin{gathered}
\frac{d^{2} \psi}{d x_{\xi}^{2}}+\left(\psi-\psi^{3}\right)-\frac{U^{2}}{\kappa^{2}} \psi=0, \\
\frac{d^{2} U}{d x_{\xi}^{2}}-\frac{\psi^{2}}{\kappa^{2}} U=0,
\end{gathered}
$$

где $\kappa=\lambda / \xi-$ параметр ГЛ, $\psi-$ нормированный параметр порядка: $\psi=\Psi / \Psi_{0}$, здесь $\Psi_{0}$ - параметр порядка в объеме сверхпроводника при нулевом внешнем магнитном поле. При этом векторный потенциал имеет лишь $y$-компоненту, $\mathbf{A}=\mathbf{e}_{y} A(x)$. Вместо размерных значений потенциала $A$, индукции поля $B$ и плотности тока $j_{s}$ в сверхпроводнике здесь введены безразмерные величины $U\left(x_{\xi}\right), b\left(x_{\xi}\right)$ и $j\left(x_{\xi}\right)$ :

$$
A=\frac{\phi_{0}}{2 \pi \kappa \xi} U, \quad B=\frac{\phi_{0}}{2 \pi \kappa \xi^{2}} b, \quad j_{S}=\frac{c \phi_{0}}{8 \pi^{2} \kappa^{3} \xi^{3}} j,
$$

где $\phi_{0}$ - квант потока.

Для уравнения (1) использовались обычные граничные условия

$$
\begin{aligned}
& \left.\frac{d \psi}{d x_{\xi}}\right|_{x_{\xi}=0}=0, \\
& \left.\frac{d \psi}{d x_{\xi}}\right|_{x_{\xi}=d}=0,
\end{aligned}
$$

где $x_{\xi}=x / \xi$ и $d=D / \xi$. Поскольку транспортный ток $I_{t}$ в пластине создает магнитное поле

$$
H_{I}=\frac{2 \pi}{c} I_{t},
$$

полное поле вблизи поверхностей пластины равно $H \pm H_{I}$, и граничные условия к уравнению (2) имеют следующий вид:

$$
\left.b\right|_{x_{\xi}=0}=h-h_{l},\left.\quad b\right|_{x_{\xi}=d}=h+h_{I},
$$

где

$$
h=\frac{H}{H_{\xi}}, h_{I}=\frac{H_{I}}{H_{\xi}}, H_{\xi}=\frac{\phi_{0}}{2 \pi \kappa^{2} \xi^{2}} .
$$

При необходимости исследования температурной зависимости критического тока $I_{c}(T)$, температура может быть учтена в модели через ее связь с $\lambda$ и $\xi$. Поскольку исследование $I_{c}(T)$ выходит за рамки данной работы, считается, что все расчеты проводятся при некоторой фиксированной температуре, близкой к критической $T_{c}$.

Для учета изменения сверхпроводящих свойств по толщине пластины вводятся зависимости $\lambda(x)$ и $\xi(x)$ (рис. 1). В рамках модели применяется квадратичный закон изменения длины когерентности по координате $x$

$$
\xi(x)=\xi_{C}\left(1-\eta\left(\frac{x}{d}-\frac{1}{2}\right)^{2}\right),
$$

где $\xi_{C}-$ значение длины когерентности в центре пластины. Такой закон объясняется зависимостью длины

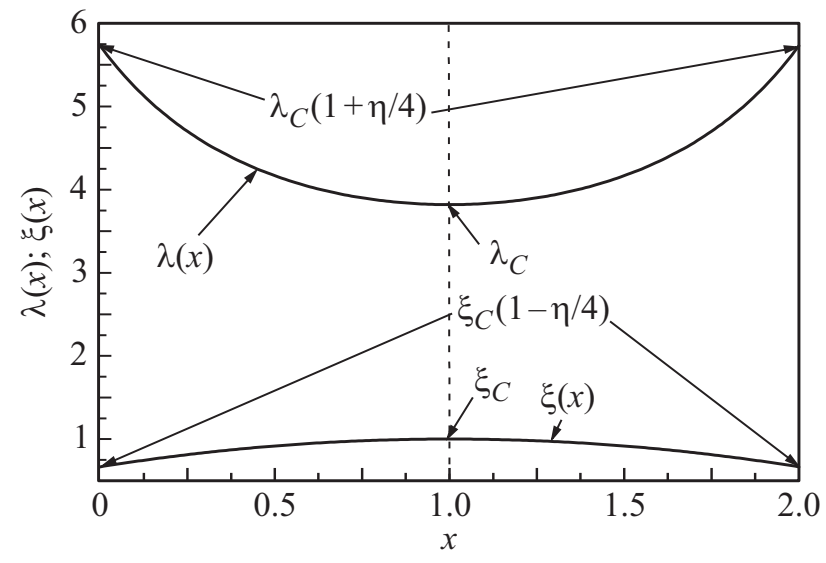

Рис. 1. Модельное распределение длины когерентности $\xi(x)$ и лондоновской глубины проникновения магнитного поля $\lambda(x)$ по толщине сверхпроводящей пластины на примере пленки с толщиной $d=2$.

когерентности от длины свободного пробега электронов в сверхпроводнике. Предполагается, что эта длина и, как следствие, длина когерентности максимальны в центре сверхпроводящей пленки и уменьшаются при приближении к ее границам. Зависимость (6) в первом приближении и учитывает эту особенность свойств реальных сверхпроводящих пленок. Распределение глубины проникновения магнитного поля $\lambda(x)$ определяется исходя из требования в рамках „грязного“ предела постоянства произведения $\lambda \xi$ по толщине пленки. В случае малого значения параметра $\eta$ для $\lambda$ также получается квадратичная зависимость от $x$

$$
\lambda(x)=\frac{\lambda_{C}}{1-\eta\left(\frac{x}{d}-\frac{1}{2}\right)^{2}} \approx \lambda_{C}\left(1+\eta\left(\frac{x}{d}-\frac{1}{2}\right)^{2}\right),
$$

где $\lambda_{C}-$ значение лондоновской глубины проникновения в центре пластины.

При этом глубина проникновения магнитного поля, наоборот, достигает минимума (равна $\lambda_{C}$ ) в центре и возрастает при приближении к ее границам. На границах пленки значения $\lambda$ и $\xi$ равны $\lambda_{C}(1+\eta / 4)$ и $\xi_{C}(1-\eta / 4)$ соответственно. Таким образом, $\eta$ - параметр, характеризующий отличие длины когерентности и лондоновской глубины в центре пленки и на ее границах. Если $\eta=0$, то выражения (6), (7) принимают вид $\lambda=\lambda_{C}$ и $\xi=\xi_{C}$, что соответствует случаю однородной пленки. Отметим, что все приведенные ниже значения длины и толщины представлены в единицах $\xi_{C}$.

Применялась следующая итерационная процедура нахождения самосогласованных решений системы уравнений (1),(2). Первоначально задавалась некоторая пробная функция параметра порядка $\psi\left(x_{\xi}\right)$ и находилось решение уравнения (2) для функции $U\left(x_{\xi}\right)$. Найденная $U\left(x_{\xi}\right)$ подставлялась затем в уравнение (1), и, с учетом граничных условий (3), находилась новая функция $\psi\left(x_{\xi}\right)$. Далее вновь решалось уравнение $(2)$, и 

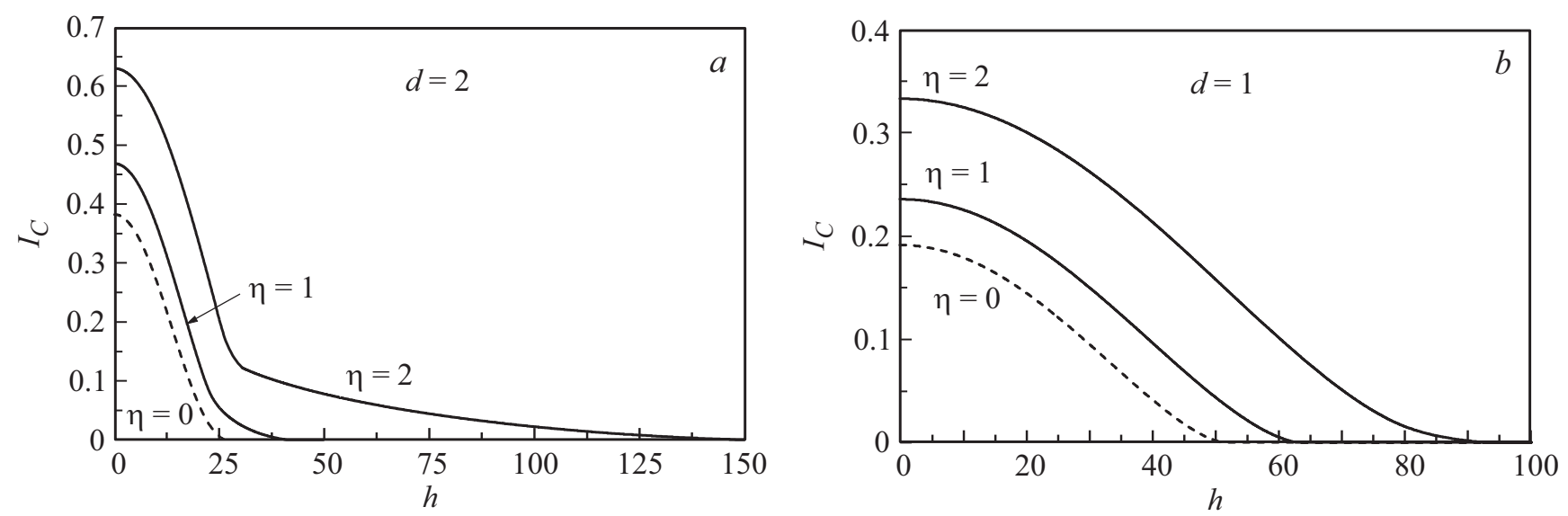

Рис. 2. Зависимости критического тока $I_{c}$ от внешнего магнитного поля $h$ для пластин различной толщины: $(a) d=2$ и $(b) d=1$. Расчеты проведены для случаев однородного $(\eta=0)$ и неоднородного $(\eta=1$ и $\eta=2)$ распределения сверхпроводящих свойств по толщине пластины.

вся процедура повторялась, пока функции $\psi\left(x_{\xi}\right)$ и $U\left(x_{\xi}\right)$ не переставали меняться и, таким образом, представляли собой самосогласованное решение системы уравнений. Найденное таким методом решение устойчиво, поскольку оно не меняется при малых первоначальных возмущениях. Значение критического тока $I_{c}$ сверхпроводящей пластины принималось равным значению транспортного тока $I_{t}$, при котором параметр порядка становился равным нулю по всей толщине пластины, $\psi\left(x_{\xi}\right)=0$. Данным методом находились зависимости критического тока $I_{c}$ от внешнего магнитного поля $h$. Отметим, что значения магнитного поля представлены в единицах $H_{\xi_{C}}(5)$ с параметром $\xi_{C}$ вместо длины когерентности $\xi$. Значения силы тока в рамках модели представляются через $H_{I}(4)$ и поэтому так же как и магнитное поле, выражены в единицах $H_{\xi_{c}}$.

\section{3. Результаты численных расчетов}

Примеры зависимостей критического тока $I_{c}$ от внешнего магнитного поля $h$ для пластин различной толщины $(d=2$ и $d=1)$ представлены на рис. $2(a, b)$. Пунктирной линией обозначены зависимости, соответствующие случаю однородного распределения сверхпроводящих свойств по толщине пленки $(\eta=0)$. Сплошная линия соответствует учету неоднородности сверхпроводящих свойств по толщине пластины $(\eta=1$ и $\eta=2)$. Расчеты для однородной пленки проведены при значении параметра ГЛ $\kappa=3.82$. Такая величина соответствует ниобию $(\mathrm{Nb})$, при этом его $\xi(0)$ равна $12.3 \mathrm{~nm}[5]$, а $\lambda(0)$ составляет $47 \mathrm{~nm}$ [6]. В случае неоднородной пленки в центре пластины принимается $\kappa=\lambda_{C} / \xi_{C}=3.82$, а при приближении к ее границам параметр ГЛ $\kappa$ возрастает в соответствии с видом зависимостей $\lambda(x)$ и $\xi(x)(6),(7)$ (см. также рис. 1). Согласно рис. 2, неоднородное распределение сверхпроводящих свойств, заданное видом $\lambda(x)$ и $\xi(x)(6),(7)$, в пленке приводит к заметному увеличению критического тока при каждом значении внешнего магнитного поля относительно случая однородного распределения сверхпроводящих свойств. При этом, чем тоньше пленка, тем сильнее относительное увеличение критического тока (сравните рис. 2, $a$ и 2,b).

На рис. 3, $а$ приведены примеры распределения параметра порядка $\psi$ по толщине сверхпроводящей пластины толщиной $d=2$ при различных значениях внешнего магнитного поля $h$. На рисунке представлено сравнение распределения $\psi$ по толщине для случаев неоднородной $(\eta=1)$ и однородной пластины. При относительно слабых магнитных полях $(h=1)$ реализуется мейснеровское состояние. При этом параметр порядка в неоднородной пластине имеет меньшее значение в ее середине и большее на краях относительно случая однородной пластины. Такое перераспределение параметра порядка связано с тем, что параметр ГЛ $\kappa$ возрастает при приближении к границе пластины, и, таким образом, сверхпроводящее состояние на ее приграничных слоях более устойчиво к подавлению током или магнитным полем, чем в центральной части. Это, в свою очередь, приводит к тому, что параметру порядку энергетически выгодно увеличиться у границ пленки и уменьшиться в ее центре. Таким образом, экранирующая способность неоднородной пластины возрастает, и ее сверхпроводящее состояние в целом более устойчиво к влиянию тока или магнитного поля. Это является причиной увеличения значения критического тока неоднородной пластины в мейснеровском состоянии. При больших полях $(h=20$ и $h=25)$ реализуется состояние, когда распределение параметра порядка $\psi(x)$ имеет следующий вид: его значение на одной из границ значительно отличается от значения на другой границе, при этом максимум параметра порядка достигается не около центра пластины, а около одной из ее границ. Данное состояние для случая неоднородной пластины имеет большее значение параметра порядка в каждой точке пластины, чем для однородной пластины при таких же условиях (внешнее 

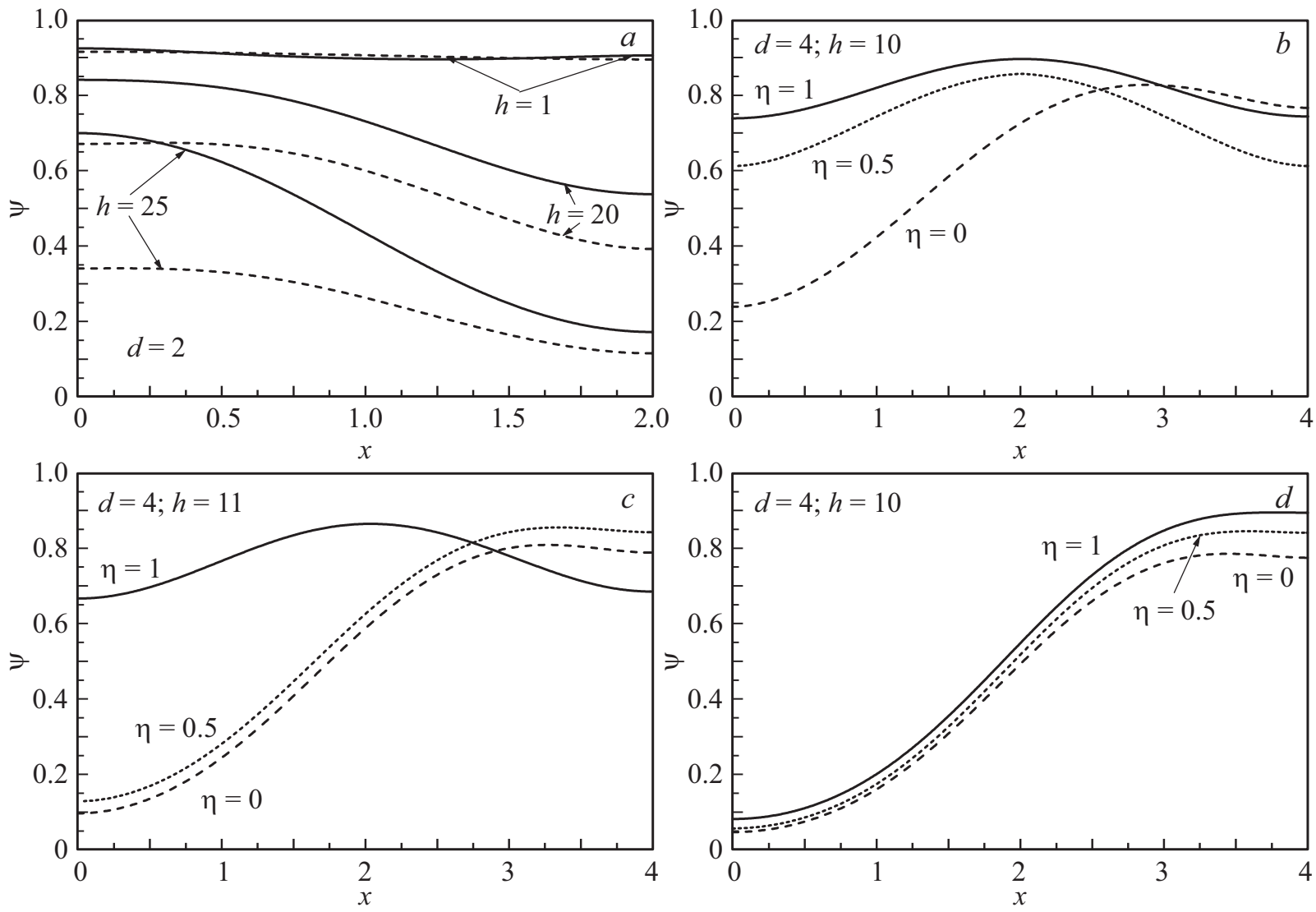

Рис. 3. Распределения параметра порядка $\psi$ по толщине сверхпроводящей пластины при различных значениях внешнего магнитного поля $h$. Расчет проведен для пластин толщиной $d=2(a)$ и $d=4(b-d)$ для случаев однородного $(\eta=0$, штриховая линия) и неоднородного ( $\eta=1$, сплошная линия и $\eta=0.5$, пунктирная линия) распределений сверхпроводящих свойств по толщине пластины. Расчет осуществлен при транспортном токе $I_{t}=0.9 I_{c}(a)$ и $I_{t}=0(b-d)$.
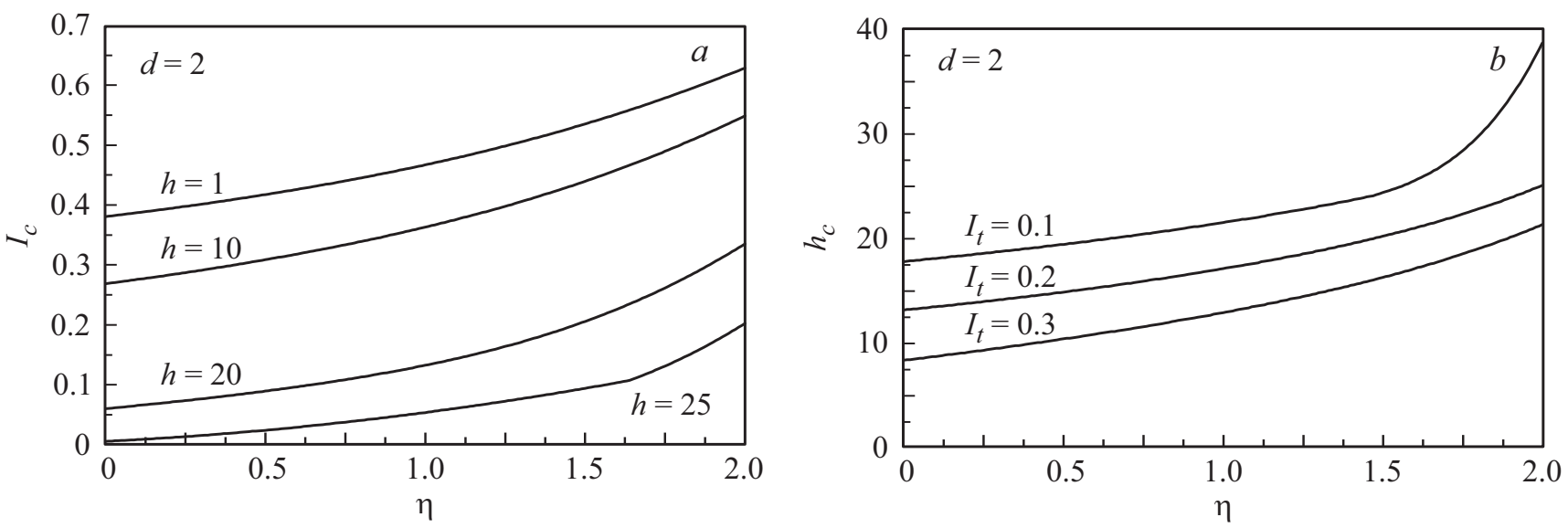

Рис. 4. Зависимости критического тока $I_{c}(a)$ и критического магнитного поля $h_{c}(b)$ от коэффициента $\eta$, характеризующего степень неоднородности пленки, для пластины толщиной $d=2$.

магнитное поле, транспортный ток) и таким образом более устойчиво к подавлению транспортным током и магнитным полем.

При отсутствии транспортного тока $\left(I_{t}=0\right)$ система уравнений (1),(2) при заданных граничных условиях (3), (5) имеет два вида решений для параметра порядка $\psi(x)$ : симметричные и асимметричные. Симметричные решения соответствуют мейснеровскому состоянию, а асимметричные - вихревому состоянию $[2,4]$. На рис. $3 b-d$ представлены распределения параметра порядка $\psi(x)$, рассчитанные для однородной и неоднородной $(\eta=0.5$ и $\eta=1)$ пластины. Распределения по- 
лучены при различных значениях внешнего магнитного поля $h$ и при отсутствии транспортного тока $\left(I_{t}=0\right)$. Перестройка параметра порядка $\psi(x)$ из вида, соответствующего мейснеровскому состоянию, в вид, соответствующий вихревому состоянию в случае неоднородной пластины происходит при бо́льших значениях внешнего магнитного поля $h$, чем для однородного случая. При этом, чем степень неоднородности выше (чем больше $\eta$ ), тем при бо́льших полях разрушается мейснеровское состояние, то есть безвихревое мейснеровское состояние сохраняется для неоднородных пластин в больших магнитных полях.

На рис. 4 представлены зависимости $I_{c}(\eta)$ (a) и $h_{c}(\eta)(b)$, рассчитанные при различных значениях внешнего магнитного поля $h$ и транспортного тока $I_{t}$ соответственно. Значения критического тока $I_{c}$ и критического магнитного поля $h_{c}$ при каждом конкретном значении внешнего магнитного поля $h$ и транспортного тока $I_{t}$ возрастают при увеличении $\eta$. Результаты расчетов показывают, что зависимости критического тока от внешнего магнитного поля для тонких сверхпроводящих пленок сохраняют качественный вид при учете их неоднородности по толщине. При этом количественно значения критического тока ощутимо изменяются. В заключение стоит отметить, что рассчитанный критический ток является оценкой сверху для реального критического тока тонких сверхпроводящих пленок. При этом данная величина дает представление о поведении других термодинамических величин, например, критического магнитного поля.

\section{4. Заключение}

Результаты нашей работы показывают, что численное решение уравнений ГЛ дает дополнительные возможности исследования критического состояния сверхпроводящих пластин. В частности, численные методы позволяют изучать влияние неоднородности пластины на ее сверхпроводящие свойства. Основные результаты данной работы можно сформулировать следующим образом:

- предложен подход к моделированию критических параметров тонких сверхпроводящих пленок, учитывающий неоднородность сверхпроводящих свойств по их толщине;

- в рамках предложенного подхода проведен расчет зависимостей критического тока от внешнего магнитного поля для пластин различной толщины. Установлено, что при заданных модельных распределениях глубины проникновения $\lambda$ и длины когерентности $\xi(6),(7)$ значения критического тока больше, чем для однородной сверхпроводящей пластины;

- проанализировано распределение параметра порядка $\psi$ по толщине пластины для случаев неоднородного и однородного распределений сверхпроводящих свойств. Показано, что параметр порядка неоднородной пластины больше и, как следствие, сверхпроводимость в неоднородной пластине более устойчива к подавлению током и магнитным полем. При этом, перегрев мейснеровского состояния неоднородной пластины сохраняется при больших полях, чем в однородной пластине. Таким образом, установлено, что чем неоднородность пластины больше, тем более устойчиво мейснеровское состояние в ней к подавлению магнитным полем;

- показано, что значения критического тока и критического магнитного поля при каждом конкретном значении внешнего магнитного поля и транспортного тока, соответственно, возрастают при увеличении $\eta$.

\section{Список литературы}

[1] Jie Yong, S. Lee, J. Jiang, C.W. Bark, J.D. Weiss, E.E. Hellstrom, D.C. Larbalestier, C.B. Eom, T.R. Lemberger. Phys. Rev. B 83, 104510 (2011).

[2] П.И. Безотосный, С.Ю. Гаврилкин, А.Н. Лыков, А.Ю. Цветков. Краткие сообщения по физике ФИАН 6, 3 (2014).

[3] П.И. Безотосный, С.Ю. Гаврилкин, А.Н. Лыков, А.Ю. Цветков. Краткие сообщения по физике ФИАН 12, 26 (2014).

[4] П.И. Безотосный, С.Ю. Гаврилкин, А.Н. Лыков, А.Ю. Цветков. ФТТ 57, 1277 (2015).

[5] J.D. McCambridge. PHD dissertation „The superconducting properties of Niobium-Titanium alloy multilayers". Yale University (1995). P. 66.

[6] В.В. Шмидт. Введение в физику сверхпроводников. МЦНМО, М. (2000). С. 36.

Редактор Ю.К. Китаев 